Western University

Scholarship@Western

Human Environments Analysis Lab (HEAL)

3-2019

Examining the Social and Built Environment Factors Influencing

Children's Independent Use of Their Neighborhoods and the Experience of Local Settings as Child-Friendly

Janet Loebach

Jason A. Gilliland 


\title{
Examining the Social and Built Environment Factors Influencing Children's Independent Use of Their Neighborhoods and the Experience of Local Settings as Child-Friendly
}

\author{
Janet Loebach' and Jason Gilliland'
}

\begin{abstract}
Neighborhoods have traditionally served as important settings for children's independent activities, but use has declined dramatically. Global positioning system (GPS) monitors, activity diaries, annotated maps, and Google Earth-enabled interviews captured the neighborhood perceptions, usage, and independent activity ranges of twenty-three children (nine to twelve years) from London, Canada. While few participants used neighborhood settings on a habitual basis, local parks and homes of nearby friends were important independent destinations. Usage was strongly influenced by positive and negative social conditions, but local environments did not generally cater well to the shifting interests of resident children. Embedding childfriendly affordances through neighborhood planning may improve children's experience and independent use of local settings.
\end{abstract}

\section{Keywords}

activity spaces, affordances, child-friendly environments, community planning, GPS, independent mobility, neighborhood use, play, urban form

Increasing recognition that children's everyday environments play a role in their health and well-being has provided the impetus for delving deeper into the spatial context of their activities, and for exploring more directly children's lived experience in their daily environments. Research has highlighted the role of the neighborhood as a critical childhood environment that helps foster the development of key skills and competencies. Traditionally, the neighborhood has served as the first setting beyond the home that children begin to independently explore, learning to negotiate the challenges of new physical environments as well as building social relationships beyond their family (Kyttä 2003; Mikkelsen and Christensen 2009; Rissotto and Giuliani 2006). As they develop both competence and confidence, children will often push the boundaries of their domains, exploring settings further and further from home (Churchman 2003; Gill 2007; Moore 1986; Spencer and Woolley 2000). Neighborhood interactions, with the skills and learning they engender, are considered by some to be key contributors to children's continued healthy development (Christian et al. 2017; Gray 2011; Rissotto and Tonucci 2002; Villanueva et al. 2016). The purpose of this study is twofold: to examine children's independent use and experience of their neighborhoods and the environmental factors that influence local activity, and to highlight the community experiences of a contemporary group of children so that they might inform environmental and policy improvements that can make neighborhoods more child- and youth-friendly.

\section{Children's Changing Neighborhood Experience?}

There is evidence that the relationship between children and their neighborhood environments has fundamentally altered during the last several decades. Studies from many Western countries have documented a dramatic decline in the time and freedom contemporary children are given to independently explore their neighborhoods (Hofferth and Sandberg 2001; Kyttä et al. 2015; Shaw et al. 2013). Increased urbanization and higher volumes of motorized traffic have been cited as contributing to decreased independent travel among children (Aarts et al. 2012; Buliung, Mitra, and Faulkner

\footnotetext{
'Western University, London, ON, Canada

Initial submission, August 2016; revised submissions, December 2017, June and October 2018; final acceptance, December 2018

Corresponding Author:

Janet Loebach, I44I Oakglen Dr., Kingsville, ON, Canada N9Y 2XI.

Email: jeloebach@gmail.com
} 
2009; Karsten 2005; Mikkelsen and Christensen 2009; Villanueva, Giles-Corti, Bulsara, Timperio, et al. 2012). The type and spatial range of outdoor activities that parents feel are sufficiently safe or socially acceptable are also diminishing, and restrictions on independent activity are extending much later into childhood than was common for previous generations (Jack 2008). A recent poll in the United Kingdom found that almost 50 percent of adults felt children under the age of fourteen should not be allowed outside without adult supervision, even when in the company of peers (Children's Society 2007); another opinion survey found that playful activities such as climbing trees, playing in local parks, or riding a bike to a neighbor's house are not condoned by many parents unless an adult is present (Jack 2008; Play England 2008). In response to anxieties about safety, many parents have begun severely limiting children's access to and activities within the public spaces of the neighborhood (Carver, Timperio, and Crawford 2008; Fyhri et al. 2011; Villanueva, Giles-Corti, Bulsara, Timperio, et al. 2012; Wilson, Clark, and Gilliland 2018; Woolley 2006).

Concerns about children's welfare in neighborhood places may also be contributing to the increasing structuring and institutionalizing of their daily activities. Gray (2011) contends that unstructured, "free play" has been steadily declining in North America since the 1950s; longitudinal studies in the United States concluded that children experienced a 25 percent decrease in time available for free play between 1981 and 1997 (Hofferth and Sandberg 2001). "Unstructured free play" outdoors has given way to more structured and privatized forms of recreation, which often takes place indoors (Active Healthy Kids Canada 2012; Burdette and Whitaker 2005a; Gray 2011; Rasmussen 2004). Karsten's (2002, 2005) work has highlighted how outdoor neighborhood play, once the dominant form of leisure activity for children, has been supplanted for many by indoor activities. Many Western children are now more likely to spend their days sequencing through a series of "protected," supervised environments, spending a significant amount of their out-of-school time in structured activities such as private lessons or organized sports (Burdette and Whitaker 2005b; Karsten 2005; Shaw et al. 2013). Aitken $(2001,124)$ suggests that parents feel they can rest easier when their children are engaged in structured, supervised activities knowing they are "safe from the perils of the streets."

These cultural and environmental shifts, coupled with dramatic reductions in the time and license children have to access local environments, suggest the neighborhood no longer serves as a common setting for children's activities. While only a small set of studies has sought to capture what are alternatively called children's "home ranges," "territories," "activity spaces" or "neighborhood domains," many of which are now more than twenty-five years old, findings to date support growing anecdotal evidence that children's local, independent geographies are significantly smaller than those of previous generations (Gaster 1991; Hillman,
Adams, and Whitelegg 1990; Karsten 2005; Kyttä et al. 2015; Pooley, Turnbull, and Adams 2005). This loss of key neighborhood experiences and interactions may have negative consequences for children's healthy development and well-being.

\section{Improving Neighborhood "Child- Friendliness"}

Creating more appealing, child-friendly neighborhood environments may help to reverse declining levels of independent outdoor activity. Riggio (2002, 45) characterizes child-friendly settings as places "where children's rights to a healthy, caring, protective, educative, stimulating, non-discriminating, inclusive, culturally rich environment are addressed." Numerous studies of children's environmental use and preferences suggest that child-friendly settings exhibit a careful balance between the protection and freedom of children; indicators of "friendliness" might then include access to attractive and safe settings that support a diverse range of activities and interactions, as well as independent movement through the neighborhood (Bjorklid and Nordstrom 2007; Chawla 2002; Kyttä 2003; Riggio 2002; Villanueva et al. 2016). Churchman (2003) also suggests that a neighborhood is child- or youth-friendly to the extent that resident children feel welcomed and equal, valued members of the community. These messages can be transmitted through the types of places the neighborhood provides for children to play and gather, safely and legitimately, as well as the welcoming attitudes and behaviors of the adult population (Churchman 2003; Horelli 2007; Villanueva et al. 2016; Woolley et al. 1999). Other studies with children and youth consistently confirm that the ability to freely gather with their friends in neighborhood public places, and the freedom to act as they choose within these spaces, are key to engendering a sense of belonging, place attachment, and legitimate dwelling (Chawla 2002; Cosco and Moore 2002; Haikkola et al. 2007; Jack 2008; Kyttä 2003; Layard and Dunn 2009; Woolley et al. 1999).

Understanding the environmental affordances that appeal to children, and which are perceived as being supportive of their interests (Gibson 1979; Kyttä 2002), can help identify features and conditions that can serve to make environments more child- and youth-friendly. Kyttä (2004) emphasizes, however, that it is not enough to perceive affordances in the neighborhood; a child must also be given the freedom and opportunity to use them through some measure of independent mobility. The ideal child-friendly environment is one where children have a high degree of freedom to explore their community, which in turn provides safe, welcoming, and diverse settings for their preferred activities.

Research-informed neighborhood planning practices and policies, particularly those that seek to engage directly with youth, can help foster such child-friendly community environments. Despite an increased call for integrating youth into 


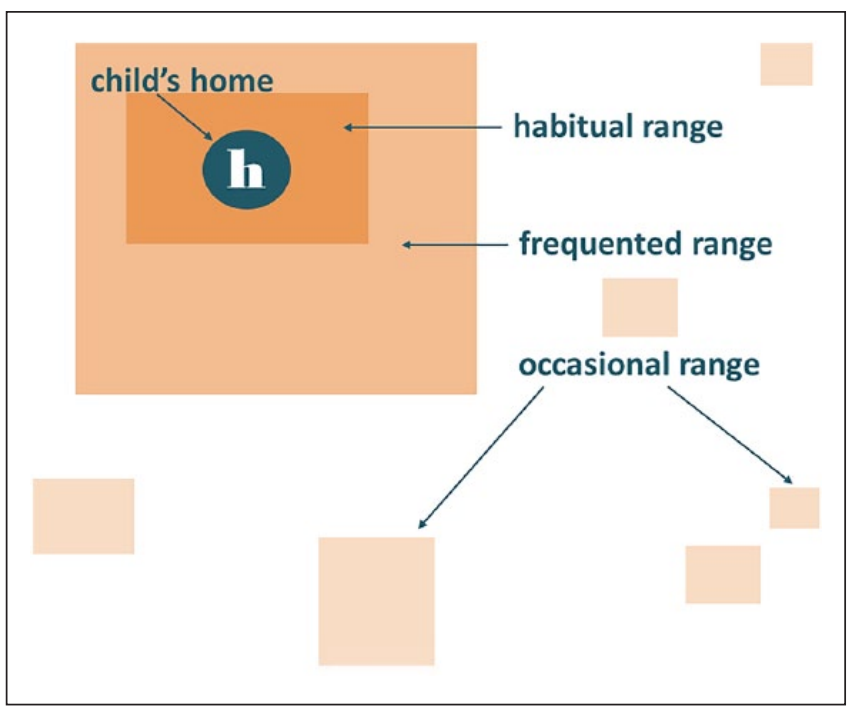

Figure I. Model of children's neighborhood activity ranges. Source: Adapted from Moore (1986).

decision-making, urban planning processes still show little systemic integration of children's voices, and youth needs remain significantly under represented in planning approaches (Bessell and Mason 2014; Osborne et al. 2017). Planners and community developers must do more to work collaboratively with youth to plan communities that bolster positive neighborhood experiences for children across the full range of childhood and adolescence. To do so, planners need a stronger evidence base around children's neighborhood activities and preferences to leverage the necessary practice and policy changes.

\section{Identifying Children's Neighborhood "Activity Spaces"}

Our understanding of contemporary children's neighborhood interactions is considerably enhanced when we can identify the environmental domains in which their activity routinely takes place. To characterize children's local "activity spaces," this study capitalizes on Moore's (1986) conceptual model of nested neighborhood activity domains to characterize the spatial and temporal differences in children's use of their local environments (see Figure 1). A child's habitual range is defined as the neighborhood environment around their home, which they use on an almost daily basis for their out-ofschool activities (Moore 1986). A child's frequented range, broader than their habitual range, includes those community spaces the child accesses periodically when granted both the necessary time and freedom. The third domain, a child's occasional range, represents the absolute edges of a child's independent territory and includes those places that a child accesses only very occasionally; it may also include places accessed via public transportation, and therefore no longer represents a strictly pedestrian territory. As children mature, and are awarded more independence, the size and nature of each of these domains usually expands to encompass more territory (Matthews 1992; Moore 1986; Villanueva, GilesCorti, Bulsara, Timperio, et al. 2012). The size of these activity ranges, however, may be highly variable among children, depending on factors such as age, gender, personality, and parental perceptions of the neighborhood (Knoll and Roe 2017; Matthews 1992; Spilsbury 2005; Valentine 1997; Villanueva, Giles-Corti, Bulsara, McCormack, et al. 2012).

As conceptualized by Moore (1986), the majority of children's daily activities would therefore take place within the nested domains of the "habitual" and "frequented ranges"; this combination represents the activity space commonly used by children independently, on foot or bicycle, in the course of their daily and weekly activity routines. Taking the more universal term activity space to be synonymous with the term domain or range, this study employs the concepts of habitual and frequented activity spaces to help visualize participant's neighborhood-based activity.

Understanding children's perception and use of their primary environments, such as the neighborhood, is key to untangling the influence of the social and built environment on children's health and development (Talen and Coffindaffer 1999). While children's neighborhood behaviors have been studied to some degree for more than fifty years, the experience of childhood has changed so drastically for many Western children that we need to know much more about their contemporary neighborhood activity, and the new cultural or environmental factors of the neighborhood that may be hampering local independent activities. This study addresses this gap by capturing children's independent neighborhood activity and examining the local social or environmental factors that may influence this behavior.

\section{Method}

The first part of the analysis in this paper employs global positioning system (GPS), interview, and activity diary data to establish children's local independent destinations and assess the extent of their neighborhood activity spaces (NAS). An intensive thematic analysis of multiple data sources is then used to confirm patterns in participant's neighborhood perception and use, and to highlight factors that may influence the size or use of their NAS.

\section{Study Participants and Settings}

This study uses data gathered for the STEAM (SpatioTemporal Environment and Activity Monitoring) project (steamproject.ca), a multiyear study designed to examine the effects of the built environment on children's health-related behaviors, including physical activity (Mitchell, Clark, and Gilliland 2016), active travel (Wilson, Clark, and Gilliland 2018), and health-related quality of life (Tillmann, Clark, and Gilliland 2018). Children aged nine to thirteen years 
were invited to participate in a week-long protocol to document their neighborhood activities, perceptions, and experiences. This study uses data from Year 1 of STEAM, which focused on two school neighborhoods in London, Canada, categorized respectively as "urban" and "suburban," which exhibit very similar socioeconomic profiles but very different built environments.

Both study neighborhoods tend toward the vulnerable end of the socioeconomic scale; families in both areas exhibit incomes that are generally lower than the city average (urban neighborhood median: \$54,244; suburban neighborhood median: $\$ 77,781$; and city median: $\$ 82,231$; City of London 2016). In addition, both areas have higher percentages of families headed by lone parents (urban: 27.4\%; suburban: 22.1\%; city: 19.2\%) and higher percentages of adult residents whose highest level of educational attainment is high school or less (urban: 46\%; suburban: $42 \%$; city: $37 \%$; City of London 2016). The two communities both have relatively stable populations, but the urban community has a higher population density (approximately 3,450 vs. 800 persons/ $\mathrm{km}^{2}$, respectively; City of London 2016).

Key informants in each community, including school principals and community support workers, suggest that students in both school neighborhoods come from a wide range of household types, including families living in social housing and many single-parent families, as well as a diverse mix of ethnicities. Informants also claim that both neighborhoods have areas that are considered "rough" or "dangerous," and grapple with issues such as community violence, gangs, or drugs. The "urban" school neighborhood, located close to the city center, has environmental features characteristic of many urban settings, including primarily a gridiron street pattern, a diverse mix of land uses, and a higher density of intersections. This neighborhood also encompasses several commercial corridors and clusters, and is fairly well served by parks and other public recreational facilities. The "suburban" school neighborhood has a meandering street pattern with cul-de-sacs and low traffic volumes common to many suburban settings, and is dominated by medium-density residential areas with scattered commercial properties or clusters and several large public parks. In addition to parks, students at the suburban school have access, within walking distance, to a substantial environmentally significant area (approximately two hundred hectares) with dense forest, wetlands, and maintained walking trails throughout. The urban school, however, has no forested areas within walking distance. These school neighborhoods have very similar socioeconomic and household conditions, yet very different built environments, allowing us to consider the impact of the neighborhood's built environment on children's local activities by holding other variables, such as socioeconomic factors, fairly constant.

Presentations outlining the goals and incentives for the study were made to older students at each school; all children in Grades 5 to 8 (aged nine to thirteen years) in each school were eligible to participate. A total of 265 students were eligible, and seventy-six (29\%) assented to participate after receiving parental consent. Child participants were given $\$ 15.00$ for each week of participation. The median age of this initial sample of participants was 11.0 years, 66 percent were female, and roughly equal numbers lived in the urban (48\%) and suburban $(52 \%)$ neighborhoods.

Participating children wore portable GPS monitors (Visiontac VGPS 900) during all waking hours for six to seven days during the spring season (May to June); GPS units marked spatial coordinates for each second of time the unit was in use. Participants also completed detailed daily activity and travel diaries, and annotated aerial photo maps of their community to highlight common destinations within the neighborhood to which they could travel independently (without an adult). Both participants and their parents also completed detailed surveys on children's neighborhood activities and perceptions.

After completing the seven-day protocol, participants were invited to take part in an optional small, interactive group interview; more than 55 percent of participants $(n=$ 42) elected to participate. A team researcher engaged participants, in groups of two or three, in sixty-minute interactive discussions about their neighborhood perceptions and behaviors. The small group format was chosen based on the team's previous experiences, which demonstrated children's greater engagement in interview discussions when accompanied by peers, and the ability of peer conversations to highlight similarities and contrasts among children's neighborhood experiences (see Loebach and Gilliland 2010). Prior to the interview, each participant's GPS tracks from their study week were loaded into Google Earth (version 2011) and then projected onto the screen in the interview room (an empty library or resource room at the study school), allowing participants to see their full week's trail of GPS tracks overlaid on a satellite image of their neighborhood; the researcher could then virtually walk through GPS trails with each child and discuss their neighborhood activities and amenities together. The tracks were explored in turn for each child in the group, and in chronological order (i.e., day by day), but the researcher allowed the children to guide much of the discussion about their own neighborhood activities. All children in the group interview were allowed to comment on each other's routes and activities, revealing where behaviors and perceptions aligned and differed. However, during the course of the interview each child was also asked a set of semistructured questions to ensure consistent information was collected from each participant. Interview comments that related to specific neighborhood resources or destinations were immediately "pinned" to their exact spatial location within Google Earth, and subsequently imported, along with their full week's set of GPS tracks, into a geographic information system (ArcGIS) to create a complete, annotated set of neighborhood activities and destinations. A transcriber was present for all interviews to capture discussions verbatim; 
however, discussions were also digitally recorded to ensure a complete and accurate transcript was available.

Only participants who completed an interview and the entire STEAM protocol $(n=37)$ were considered for inclusion in this paper. GPS data minimums, however, were also established to ensure typical patterns of behavior could be discerned; inclusion in this analysis required a minimum of three hours of GPS data on at least two weekdays and a minimum of four hours of GPS data on at least one weekend day (see Loebach and Gilliland 2016a for rationale on inclusion criteria and the reliability of the GPS data). Most participants who met these criteria far exceeded the minimums; the median recorded GPS data (during all out-of-school time) for these children was 39.0 hours per week. The final sample for this analysis $(n=23 ; 62 \%$ of those who completed both protocol and interview) had the same median age (11.0 years) as the initial sample, and similar proportions of urban $(n=$ $12)$ versus suburban $(n=11)$ students, but a higher proportion $(82 \%)$ of female participants.

\section{Establishing Independent Destinations and Domains}

Each child's pedestrian-based "neighborhood activity space" (NAS) was established within a GIS in a previous study using each participant's objectively measured GPS tracks (see Loebach and Gilliland 2016a, 2016b). All independent destinations on participants' annotated neighborhood maps or noted during interviews were added to the NAS dataset. ArcGIS' Tracking Analyst tool was also used to trace through participants GPS datasets, checking for congruency with activities and locations noted in activity diaries, verifying the relative amount of time spent in noted activities and settings, and adding any undocumented independent destinations into the GIS dataset. Each identified destination was also categorized by type, such as friend's house, park/playground, malls or other retail, and wooded/natural areas (see Table 1 for full list of destination types). This combined dataset illustrated each child's typical neighborhood use, highlighted prominent destinations and activity settings, and allowed general patterns of use across and within neighborhood environments to be examined. See Figure 2 for an example of a child's neighborhood map with GPS tracks, independent destinations, and interview comments.

The second part of the analysis involved thematic coding of interview narratives and activity diaries to clarify children's habitual patterns of neighborhood use. Narratives also highlighted the degree to which the participants experience their neighborhoods as child-friendly, and supportive of their preferred activities. Previous work examining children's neighborhood perceptions (Loebach and Gilliland 2010) provided an initial thematic framework, which was then supplemented by characteristics of child-friendly environments gleaned from the literature (Bjorklid and Nordstrom 2007; Chawla 2002; Horelli 2007; Jack 2008; Kyttä 2003, 2004).
Table I. Children's Independent Neighborhood Destinations.

\begin{tabular}{lc}
\hline Independent destination by type & $\begin{array}{c}\text { \% of total } \\
\text { destinations }\end{array}$ \\
\hline Friend's houses $^{\mathrm{a}}$ & 21.2 \\
Parks/playgrounds $^{\mathrm{a}}$ & 19.7 \\
Streets/cul-de-sacs $^{\mathrm{a}}$ & 10.1 \\
Variety stores $^{\mathrm{a}}$ & 8.2 \\
Home locations (back or front yard; common space) $^{\mathrm{a}}$ & 6.7 \\
Malls or other retail (department or drug store) $^{\text {Wooded/natural areas (including ponds, rivers, }}$ & 6.3 \\
forests, ravines) $_{\text {Multi-use trail/path }}^{\mathrm{a}}$ & 5.3 \\
Dollar/thrift stores & \\
Coffee shop/cafe & 4.8 \\
Fast food restaurants & 3.8 \\
Relative's houses & 3.4 \\
Grocery stores & 3.4 \\
Video stores & 2.4 \\
Libraries & 1.4 \\
Churches & 1.0 \\
Community center & 0.5 \\
Outdoor swimming pool & 0.5 \\
Lesson/class locations & 0.5 \\
\hline
\end{tabular}

${ }^{\mathrm{a}}$ Destinations included in habitual domains.

Each child's narrative was explored first with the preliminary thematic framework; additional themes were added to reflect new concepts that arose during reviews of the interviews. The narratives were then explored again through the lens of the updated thematic framework to ensure all comments from the narratives could be represented by one or more thematic categories (see Table 2 for the final thematic framework).

\section{Results}

\section{Children's Independent Neighborhood Domains and Destinations}

Analysis of children's GPS data, hand-drawn maps, and interview narratives together revealed a diverse range of local settings to which the children have independent access. Every identified destination was categorized by type, and the total proportion of each type (across all participants) was calculated (see Table 1). The most frequently noted destinations were nearby friend's homes and local parks or playgrounds, each comprising about one-fifth of all independent destinations. Local streets and settings around the home, along with commercial outlets such as variety stores and malls, were among the next most common destinations overall. The proportion of children listing friend's homes as a frequent destination was the same among both urban and suburban participants, but urban children mentioned a slightly higher proportion of commercial destinations, while suburban children mentioned a slightly higher proportion of recreational activity destinations such as parks. The remaining independent destinations included an array of formal and informal 


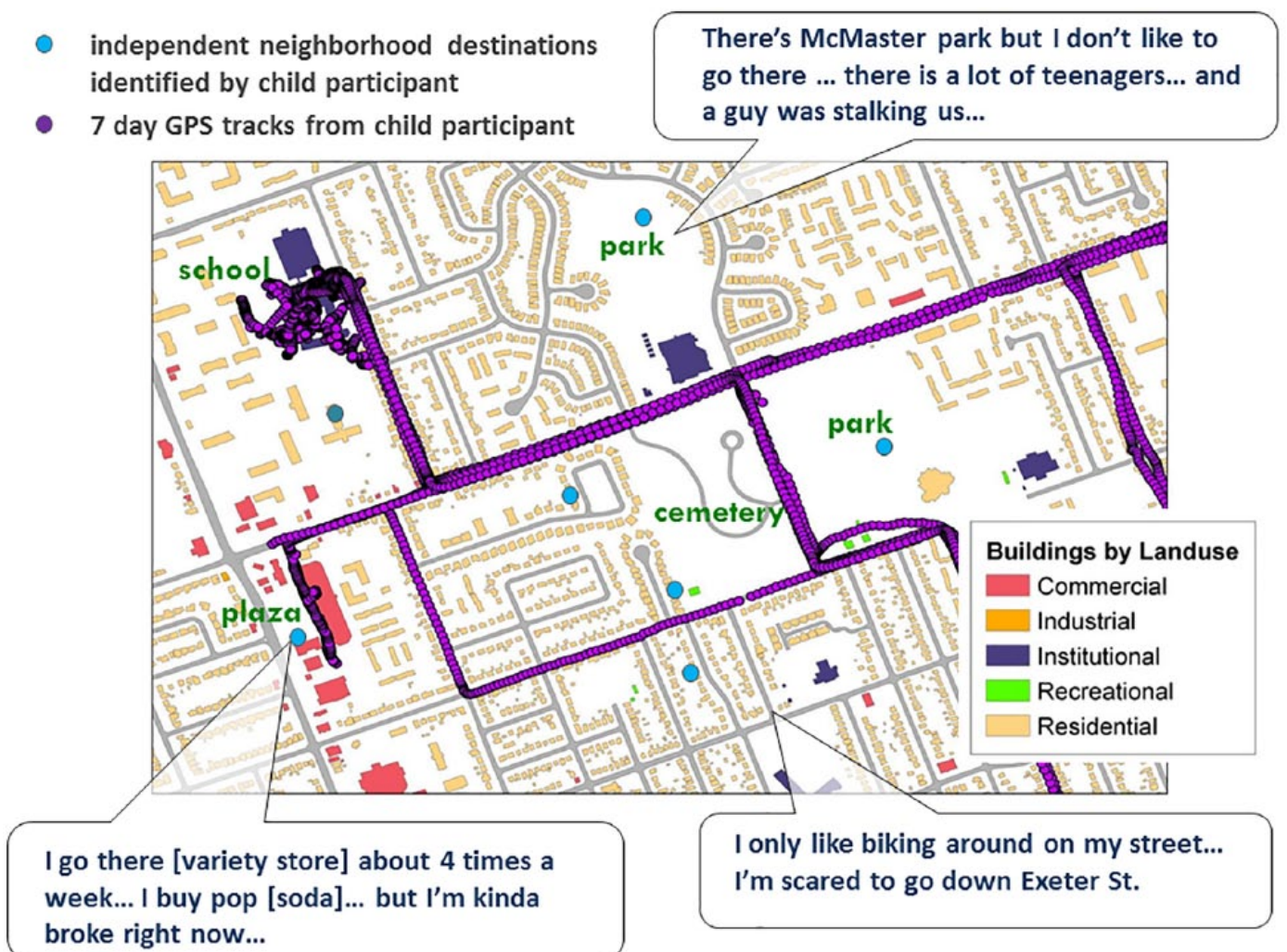

Note: GPS tracks and comments have been anonymized to protect the identity of child participants

Figure 2. Example of child participant's neighborhood map highlighting GPS tracks, child-noted independent destinations, and interview comments.

GPS = global positioning system.

Table 2. Thematic Analysis Framework.

\begin{tabular}{l}
\hline Thematic analysis framework \\
\hline i. Neighborhood perceptions regarding \\
Access to resources \\
Ease of mobility \\
Environmental supportiveness \\
Safety \\
Neighborhood identity and culture \\
ii. Neighborhood activities and destinations \\
Preferences \\
Social conditions \\
Shifting interests \\
iii. Activity facilitators or restrictors \\
Parents \\
Peers \\
Other community members \\
Social environment or circumstances \\
Built environment
\end{tabular}

recreational settings such as ponds, multi-use paths, and community centers, as well as other retail and food outlets (e.g., fast food restaurants and thrift stores), homes of relatives, and a few institutional settings such as churches or community centers. While wooded/natural destinations only made up about 5 percent of the total mentions, all but one of these mentions were from suburban children.

A relatively large number of participants $(40 \%)$ visited or noted destinations quite far from their homes (e.g., between 1 and $2 \mathrm{~km}$ away), and defined fairly large independent frequented domains. There were, however, several children whose NAS were very small and compact, usually encompassing only their yard and the street immediately in front of their home (e.g., less than $100 \mathrm{~m}$ away). There appeared to be no systemic pattern in NAS size by age, but children at the older end of the spectrum tended to note more independent destinations and spend more time in settings further from home. In line with expectations, the oldest children noted the largest number of local independent destinations; the average number of destinations for nine- and ten-year-olds was 2.7, eleven-year-old participants averaged 3.8 destinations, and the twelve-year-olds identified 8.0 independent neighborhood destinations on average. Comparing across neighborhood types, children from the suburban school frequented an average of 5.6 local destinations, slightly more than those from the urban neighborhood who averaged 4.4 locations. However, 


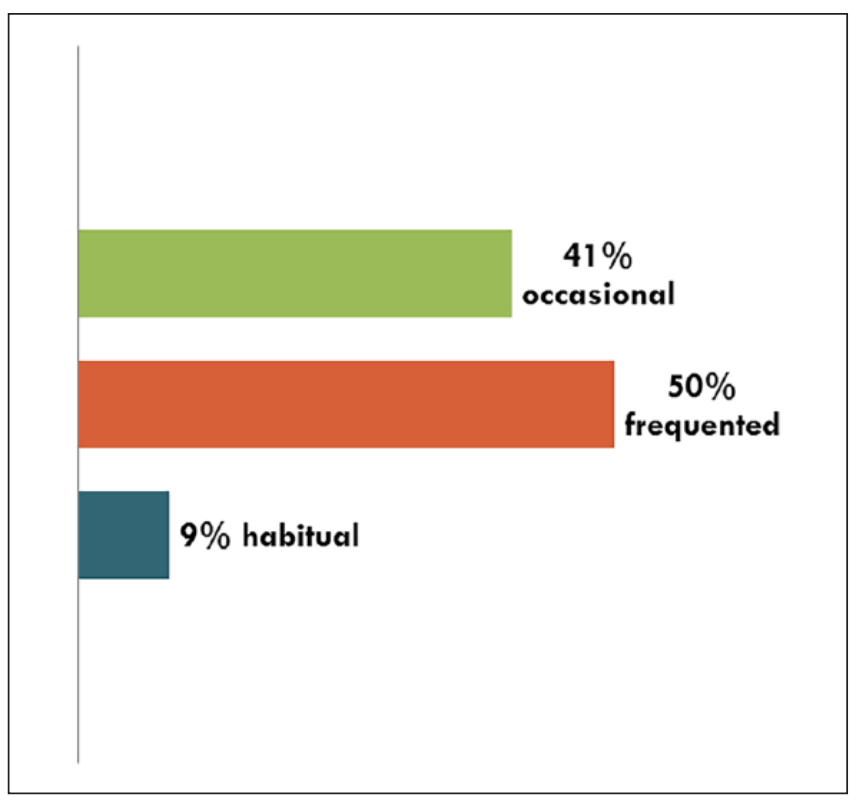

Figure 3. Proportion of independent destinations in neighborhood activity ranges by frequency of use.

more children from the suburban neighborhood $(27 \%)$ than the urban community $(8 \%)$ identified only a single destination, which they could visit independently. The two children with the highest numbers of neighborhood destinations were from the urban (fourteen locations) and suburban (eleven locations) schools, respectively, though both children were among the oldest group (twelve-year-olds). There was little overall difference in the quantity of noted destinations in the urban versus the suburban neighborhood; rather, the age of the child appeared to be a stronger influence on the number of frequented neighborhood destinations. Most of the participants in the final sample were female, precluding an accurate depiction of neighborhood activities according to gender.

Child narratives clarified not only the type and location of independent destinations but also the general frequency with which these settings were used, helping to establish the extents of the children's habitual versus frequented and occasional activity spaces. Narratives revealed that few of the destinations (only 9\%) comprised part of any of the children's habitual activity spaces (Table 1 and Figure 3). Only parks, local streets, variety stores, outdoor home locations, and multi-use trails were used by some children on an almost daily basis, but habitual use of these neighborhood settings was only found among a small proportion of participants; there was no significant difference in the habitual activity spaces of urban versus suburban participants. GPS data from the study week similarly found that participants spent 94 percent of their out-of-school time either indoors at home or outdoors within $150 \mathrm{~m}$ of their house; urban participants averaged 95 percent of their leisure time, and suburban child participants averaged 92 percent of their leisure time either indoors or outdoors at home. The majority of the independent destinations noted or visited were more likely to be part of a child's frequented (50\%) or occasional (41\%) activity space. Many children (70\%) documented their ability to travel independently to a wide range of local destinations, but most, regardless of neighborhood type, did not regularly use these community settings. A deeper analysis of intensity of use of these destinations was beyond the scope of this study.

\section{Thematic Analysis of Children's Neighborhood Perceptions and Activities}

Results from the first part of the analysis suggest that participant children, in general, are using only a small portion of their neighborhood spaces or amenities on a regular or habitual basis. Thematic coding was used to better understand the nature of children's local activities and preferences, and the factors that may be limiting local play and mobility.

The social foundation of children's neighborhood activities. The most striking characteristic of participants' neighborhood behavior was the highly social nature of both their activities and travel. Almost without exception, independent local activities primarily took place in the company of other children. Travel around the neighborhood was usually accompanied by a sibling, or friends who lived nearby; even solo journeys were often designed to meet up with friends at some local setting. The presence of other children seemed to be a key factor in making parents more comfortable with independent neighborhood activity and in some cases helping to extend the child's independent domain. When describing their activity space boundaries, many children noted access to fairly large territories "as long as I'm with a friend," or noted that they were allowed to go further with a sibling or friend than they would be allowed on their own.

Interviewing children in small groups also highlighted the extremely intertwined nature of many of their neighborhood activities. Even when addressing the activities of just one child, the recounting of stories and activities was often carried out by several members of the group, and revealed not only the social nature of their activities but also that many activities involved a shared use of local settings, as well as collective memories and knowledge of local places.

Preferred settings and shifting interests. Recreational sites in both the urban and suburban neighborhood were highlighted as valued and frequent destinations, even though participants did not necessarily spend a large proportion of their time in these settings. Parks and playgrounds very often served as the common meeting place for friends living in the same neighborhood, and featured as prominent locations for "hanging out," talking, and general socializing. Play equipment in parks such as climbers, slides, and swings are often assumed to be the primary attraction for children of all ages, but analyses underlined the shift in children's interests as 
they mature, and subsequently in the environmental opportunities they use. Visiting parks to use play equipment was now often related to the interests of younger siblings rather than their own; when Erica ${ }^{1}$ was asked why she visits a nearby park so often, she admits it is "cause my [younger] sister drags me [there]."

The children in this cohort, most of whom were at the older end of middle childhood, noted that they were "kinda done with play structures"; instead, play equipment now often served as the platform for talking and "just hanging out," or for the occasional group game. Inhabiting swings and climbers for passive socializing rather than active play may be in part due to an unsupportive environment; several children bemoaned the fact that most playgrounds and parks afforded few other places, besides on the ground, where they could gather in a group to sit and talk.

Play equipment did hold some appeal for older children, but their enthusiasm was generally reserved for more complex and challenging equipment such as zip lines, or "really, really, really tall slides," that is, equipment that better matched their more advanced capabilities and still afforded enough exciting challenge. Several children noted a preference for playgrounds outside their neighborhood that included such equipment, and to which they would ask to be driven. Local parks that primarily feature less advanced play equipment offer fewer affordances for older children whose activity interests have shifted, as this discussion with three 10-year old participants illustrates:

Richard: 'Cause the park's not really fun for us ... 'cause we're not going to, like, go down the slide or anything [laughs].

Researcher: So you think the stuff at the park is more for younger children?

Richard: Yeah, we did that when we were little...

Researcher: Is there anything they could put in the park that would make you want to go to the park?

Brad: Yeah, like zip lines!

Researcher: So if there was something that was more challenging ... something you could climb ...?

Brad: Yeah, cooler!

Colin: Yeah, something cooler that you could grab on to and slide down ... so fun!

Child narratives revealed that other simple yet flexible environmental affordances could also draw their interest and serve as neighborhood activity nodes. In the suburban school neighborhood, all nearby participants, without exception, highlighted the man-made retention pond at one end of the subdivision as a popular destination. The frequency with which children visited this area varied, particularly with proximity to it, but it was highly valued as a local resource by all. One of the older girls outlines her interest in the pond:

Kelly: I like to go there a lot because ... they have, like, in the water these sewers [drainage pipes] with the rocks all around and you can sit up top there ... and it's so relaxing to sit with your friends ... and we like to climb it.

A younger boy explains the appeal of the pond for him and his friends:

Researcher: Why do you like heading [to the pond]?

Brady: Because you can, like, skip rocks and that ... and then just hang out there. And then there's a path up here [points out path on screen] . . a little path where you can go down on a pile of rocks. Well, sometimes you may see other things... like, you may see a fish ... or may see, like, a frog or something... wildlife.

A simple pile of boulders around a drainage pipe jutting out into a small retention pond afforded the opportunity to climb up, to sit and talk, as well as a place to watch the water and local wildlife, making this an appealing activity and retreat destination for children living nearby.

Commercial destinations and the call of the mall. Children's shifting interests were most apparent among the oldest of the participants. A number indicated that they spend a lot of their free time now "hanging out" at the mall with friends, often being dropped off by parents early in the day and staying until they are picked up again hours later.

Emily: Um, Maidstone [Mall] ... I go there almost every weekend just to hang out with people.

Researcher: Do you usually go there together or you meet there?

Emily and Victoria [unison]: We go there together.

Researcher: What do you do there?

Victoria: Usually we first go to the movies 'cause it's right in the mall ... and after, sometimes ... well, occasionally, we've bought stuff. We'll just walk around and look at things.

Another older girl recounts similar mall-based activity:

Alison: I go to the mall every weekend.

Researcher: And that's with your friends?

Alison: Yeah ... I [go] with my mom . . like, my mom drops us off and then, like, in three hours she picks me up.

Researcher: So, do you just hang around or are you shopping the whole time?

Alison: We walk around and look at stuff.

Rather than spending time in their local environments as many did when they were younger, these older children preferred the opportunities available at local shopping centers. In addition to supporting their social, entertainment, and consumer interests, malls are providing an indoor destination where they can hang out with friends, regardless of weather conditions, without being under the direct supervision of parents. 
Commercial outlets in general were common destinations. Convenience stores in particular were highlighted as a popular and frequent destination of the vast majority of participants, in some cases forming part of the child's habitual activity space. Primarily valued for their provision of "treats!" such as pop (soda), chips, and candy, variety stores also figured as destinations where parents would send them on their own to run errands, such as buying household provisions.

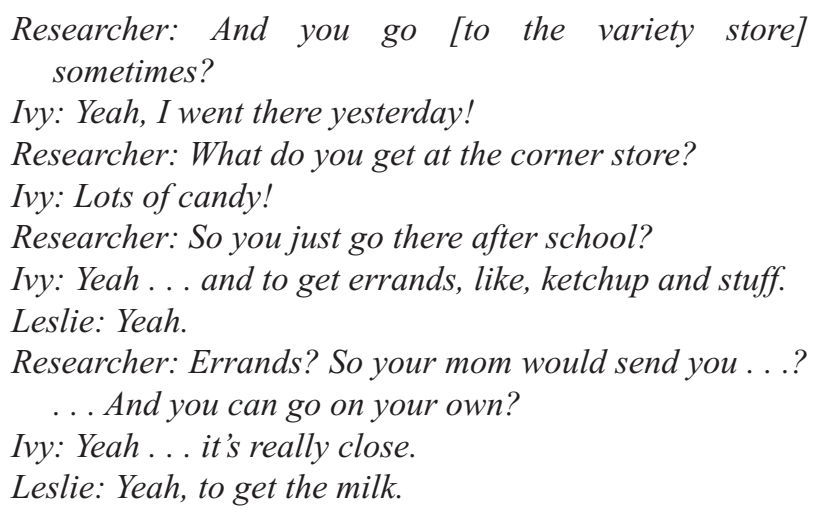

Retail outlets such as variety, dollar, and grocery stores offer a range of inexpensive food, toys, school, and craft materials that are often affordable even on a child's allowance; when located within reasonable proximity to children's homes, these stores were included in many children's frequented, if not habitual, activity spaces. Nearby stores can serve as important opportunities for independent neighborhood mobility, providing appealing activities and products that entice children to make the journey while close enough that parents feel comfortable letting children go on their own.

Unfriendly people and places. Participants were also clearly aware of potential dangers or threats in their local environments. When asked about places in their neighborhood where they felt uncomfortable or threatened, many identified a location or condition that caused them concern. These mentions were primarily related to social rather than built environment conditions. Many children expressed fears related to a specific person or group in the neighborhood whom they considered to be "scary or weird"; often this was an aggressive person or group who would yell at them or drive them away from local spaces. Brad's experience with one such neighbor limits his use of local settings:

Researcher: [What] would you say is your least favourite thing about your neighborhood? Like, ... if there was one thing you could change, what would it be?

Brad: Um . . people. . . some people are mean in my neighborhood.

Researcher: Yeah? How are they mean?

Brad: I'm trying to go in, like, this parking lot ... and some people say, like: "Get out, this is our place."
They live in this one house. . . and they're sometimes saying "get out" when I go off this way [indicating a common green space near his house].

Researcher: So, you don't feel comfortable hanging around back there because...

Brad: [interrupts] Yeah, I just don't go there.

Some children mentioned concerns about neighborhood adults, but the bulk of their discomfort was related to the presence of older teens in local public areas, particularly parks and playgrounds. Some children recounted being yelled at by older teens or told to leave the area, but confrontations or the experience of threats were actually rare; in most cases, the mere presence, or even rumoured presence, of a group of older youth was intimidating enough to make them leave the area or avoid it all together. Two female participants recount such an experience:

Researcher: So ... for the kids around your neighborhood, are there places that you can play near to your houses?

Sheila: We go to Highland [public school playground]

Darlene: Oh, yeah, Highland. We play in the playground .... I usually have to go with friends because there's some issues there ... with some ... people there. [. . .] Sometimes we can't go there because there's these people that hang out by the portables....

Sheila: Yeah, they're bad guys.

Darlene: Bad guys!

Researcher: So, they're just there sometimes? Is it at night?

Sheila: Yeah, just sometimes. At night usually. But one time I went with my friends . . . and there was this one guy on a motor bike and he was, like, running up to us really close....

Researcher: Was he joking around?

Sheila: I don't know... but he looked kinda mean.

Darlene: I get scared of people...

Sheila: ... so then we left.

The presence of older youth appeared to be a concern primarily in the evenings, and may contribute to the general perception that the neighborhood is unsafe at night. Most children in both neighborhoods generally felt safe frequenting these spaces during the day, especially when in the presence of friends. Adults who limit or discourage play behavior are often cited as the bigger threat to children's experience of public spaces as child-friendly (Collins and Kearns 2001; Jack 2008; Valentine 1996), but for most participants here, the presence of older youth was the more common barrier. Ironically, the children did not recognize that their own group presence in local parks, for example, would likely be intimidating to younger children hoping to use the same space.

Given the socially-based nature of their neighborhood anxieties, it is not surprising that the most prominent call 
for change to local environments related almost exclusively to other inhabitants. A few comments, however, suggest that the local built environment may also be a barrier to their preferred activities. Most children, for example, acknowledged that they often sit on play equipment to gather and talk with friends, but only a few explicitly recognized that there are few other places in the neighborhood that allow for them to gather or socialize in a group. Emily, bemoaning the "boring" plaza she and friends regularly walk to, explains that there are few other affordances for socializing available: "I wish there was more . . like, places to go just to hang out. Not, like, shop-wise but just places to sit ... and talk." Richard and Colin also lamented the lack of local facilities for social activities; when asked what other amenities they would like to see in their neighborhood, they tried to communicate this wish for social activity space:

Richard: I would also like someplace that's, like ... I don't know, like, cool ... like, a clubhouse for, like, all the kids ... or something....

Researcher: Like one you get to go build? Or where you ...

Colin: [interrupts] No, where you just get to hang out ... . Richard: No, just like ... sorta like the Boys and Girls club ... [...] but in our neighborhood, not all the way downtown... 'cause we're, like, probably never going to go there [to the downtown Boys and Girls club].

These youth had trouble articulating this desire because they have not experienced many places that meet these social needs; they have only a vague notion that there could be something better, or more supportive. The issue of proximity to resources was also clear, highlighting children's difficulty in accessing amenities that are far from home, and out of walking distance. Several children mentioned that they wished certain destinations were closer to home. Analysis of neighborhood use suggests that children living closer to amenities, regardless of neighborhood type, tend to use them more frequently than those further away, even when still within walking or cycling distance (as judged by the children themselves, but typically within fifteen to thirty minutes by foot or bike).

The experience of child-friendly neighborhoods?. Most indicators of child-friendly environments include the provision of safe, welcoming community environments that provide a diverse and stimulating range of opportunities for activity and interaction; children should feel that they are valued members of the community and have the freedom to legitimately play in and travel through the neighborhood environment. In the strictest sense of these terms, the majority of the children in this study experience their neighborhoods as relatively child-friendly. Participants generally perceive their neighborhoods as safe places that offer a respectable range of recreational opportunities, and feel their community is fairly easy to move around. In both the urban and suburban neighborhood, many children had some peers close by with whom to play and socialize, and most had at least one nearby place where they could safely meet. Many of the participants are given some degree of independence by their parents, which allows them to use the neighborhood amenities at their disposal.

The narratives, however, suggest that most children in the study are only engaging with their local settings in relatively limited or passive ways, regardless of their urban or suburban location. Discussions around neighborhood activities revealed few instances of creative use or shaping of the environment for activity, and little overall enthusiasm for the amenities available. Children often use the opportunities available close to home, but express little eagerness about neighborhood settings, or their activities within. Many participants' neighborhood activities were also passive and relatively sedentary; they primarily walked around with or sat and talked with friends. Occasionally, they may play "cheerleading" or a group game of tag, but these activities seemed to be played in spite of, not inspired by, the local environment. While participants did not necessarily experience their neighborhoods as $u n$-friendly, neither the urban nor suburban youth demonstrated the strong attachment to or dynamic engagement with their local settings we would expect in a genuinely child-friendly neighborhood.

Despite some differences in the spatial layout of the two neighborhoods, and their recreational, natural, and commercial amenities, there was little overall difference in how participants experienced their neighborhoods, with the exception of those children who had diverse and ample destinations within a very short distance of home. In this study, the "urban" versus "suburban" categorization proved inadequate to describe differences in the neighborhood environment; for example, despite an "urban" categorization, some students in the urban school lived in quite homogeneous residential areas with few commercial or recreational spaces nearby. Their neighborhood had a substantial sidewalk network, but there were few appealing destinations nearby to walk to. Conversely, some of the children in one area of the "suburban" neighborhood had plentiful commercial and green spaces nearby, as well as sufficient sidewalks and paths to aid mobility. This study highlights that assessments of children's neighborhood environments and associated resources must move beyond urban versus suburban characterizations and be considered at a very fine scale, particularly as many contemporary children are habitually found within a very small domain around their homes.

\section{Conclusion}

Examining the habitual destinations and activities of participating children provided several key insights about contemporary neighborhood perceptions and use, including factors that can promote or restrict local activity. First, the 
detailed analysis of children's independent neighborhood destinations and activity spaces illustrated that most participating children do not habitually spend time playing independently in their neighborhood environments, particularly in locations farther from home. Many children clearly had permission to travel to farther destinations, particularly in the company of friends or siblings, yet most of these were strictly occasional outings. The habitual NAS of participants in this study were generally very small and primarily comprised the environment within and immediately surrounding the home. The biggest reductions in neighborhood use, therefore, may be in the size of children's habitual domains, that is, the extent of the neighborhood environment they use on an almost daily basis.

The thematic analysis also highlighted that children's neighborhood activities are highly tied to nearby social conditions. Social activities dominated the list of the children's preferred activities, and local activities were almost exclusively carried out in the company of friends or siblings. Localized social conditions also negatively affected children's neighborhood activities, particularly interactions with aggressive neighbors or the intimidating presence of older youth. The availability or lack of affordances for safe social activities affected their impressions of the neighborhood and its degree of child-friendliness. Local environments that provide numerous or centrally located meeting places or "activity nodes," and safe, comfortable facilities for "hanging out" and talking with friends, could strengthen the appeal and use of neighborhood environments. The popularity of recreational and commercial settings also reinforces the importance of these resources as neighborhood nodes for children's leisure and social activities, which may help draw children away from sedentary activities indoors at home. The prevalence of local streets, paths, and trails in narratives and on both urban and suburban neighborhood maps also suggest that these pathways are not only important as connections for youth between local destinations but as appealing activity locations in their own right.

While interesting destinations at further flung distances in the neighborhood may draw children, particularly older children, deeper into their communities to experience settings further from home, it is likely for now that children's lack of independent mobility or time (as restricted by their parents or busy schedules) means that they may only be able to habitually experience settings very near to home. Contemporary planning practices and policies should, therefore, aim to provide child-friendly spaces and routes throughout communities, spread equitably through residential areas, not just, for example, in the form of large neighborhood or regional parks. This means implementing a city-wide framework that assesses all neighborhood environments and related policies for child-friendliness and that insists all development projects be examined for barriers to local children's mobility and activity at a fairly fine environmental scale. This should include deliberate provision of appealing destinations and opportunities for children of all ages within close proximity to any given home, but particularly children in middle childhood (approximately nine to thirteen years of age) who have become a forgotten public interest group.

This crucial time of social and environmental exploration is best supported by communities that offer a variety of mixed-use destinations within a five- to ten-minute walk from home, including plentiful provision of recreational and green spaces, neighborhood commercial zones, and ample networks of safe sidewalks, trails, and walking/cycling paths to increase the accessibility of local destinations. Communities, including their policies and bylaws, must also emphasize safe and welcoming public and natural spaces for youth to gather comfortably to socialize with friends, or provide more challenging recreational opportunities. The perception of appealing affordances for activity could be increased by providing amenities that provide positive challenges, such as higher "mountains" to climb or more advanced play equipment, particularly for older children whose interests and capabilities have surpassed simpler play facilities.

Lack of stimulating interactions may be impeding children's development of a relationship with their local environment. Few of the participants displayed any strong sense of attachment to their neighborhood, or a sense of belonging to their local place. They used little to no proprietary language when discussing local settings, and though they raised few complaints, there was also little expressed passion for their places. When pressed to consider neighborhood improvements they would like to see, or amenities they would wish to see installed, very few could think of specific suggestions for changes or additional resources. The children did not explicitly feel unwelcomed or unsupported in their neighborhood, but it seemed they have become accustomed to only see, and expect, a narrow range of opportunities for neighborhood activity. They could not envision these environments could be made to be more appealing and supportive of their activities. The local built environment, rather than just meeting the basic needs of these youth, should also aim to inspire and engage them, and create opportunities for cultivating place identity and belonging.

\section{Creating Affordance-Rich, Child-Friendly Environments}

This comprehensive analysis of children's independent neighborhood activity exposes a number of attributes that influence children's perception and use of local environments, and the development of local independent activity spaces. Neighborhood studies like this help to provide a valuable evidence base from which communities and urban planners can work to create more affordance-rich environments for children. This study, in line with other studies of neighborhood preferences, suggests that planners can help create more child-friendly communities by supporting conditions that expand children's activity and mobility 
opportunities, such as smaller but more densely positioned green spaces, and walking/cycling networks, as well as limiting or eliminating those planning initiatives that hinder neighborhood activity and travel for youth, such as singleuse zoning and reliance on large neighborhood- or regionalscale parks and playgrounds for play- and green space. Findings from this study were fed back to local planners through the Chief Planner of the City of London, who was a co-investigator on the project, to help inform ongoing planning initiatives in the city.

The risk-averse discourse that currently defines what is safe and normative behavior for children, and which has led some parents to severely reduce or eliminate unsupervised activity and travel, has eclipsed the evidence that reinforces the developmental benefits children receive when they tackle the challenges of the neighborhood environment (Gill 2007; Karsten 2002; MacDougall, Schiller, and Darbyshire 2009; Valentine and McKendrick 1997). Attempting to eliminate all risk by limiting activity to structured, supervised conditions may, in fact, undermine children's skill and knowledge development (Gill 2007; Mikkelsen and Christensen 2009; Shaw et al. 2013). Communities and planners also tend to operate through a framework designed to limit risk, to avoid issues of liability and potential for property damage; however, this approach can also minimize the appeal of local resources for children, and suppress their neighborhood placemaking opportunities.

A child-friendliness framework can be a useful tool for evaluating neighborhood environments and assessing the degree to which they support the healthy activities of children. Such a framework considers environmental as well as socio-cultural influences on neighborhood activity and attempts to strike a balance between providing safe yet stimulating and diverse opportunities for children. The thematic framework presented here could serve as a valuable starting point for planners seeking to engage local youth in discussions about their neighborhood experiences and preferences. Community planning and policy initiatives can use such a framework to provide neighborhood environments with a dense network of diverse amenities and graduated challenges that can help children of all ages develop and thrive. While such a framework can help to identify priority areas around which to organize these discussions, complementary participatory processes and tools also need to be tailored to address the local context and culture, as well as the needs or capabilities of the participants. To support the methodology outlined here, there are several other resources that suggest ways to effectively engage children and youth in planning processes and recognize their diverse community needs (see Bishop and Corkery 2017; Derr, Chawla, and Mintzer 2018; Driskell 2002; Gleeson and Sipe 2006; Knoll and Roe 2017). The most important takeaway is that planners actively embed genuine opportunities for engagement and discussion with youth, particularly children aged nine to thirteen years as well as older teens, around their local community needs and preferences. The process must, however, go far beyond tokenistic gestures of engagement; young citizens must be able to see that their needs and interests are eventually reflected in the planning of their communities, and that they feel welcome and safe in their neighborhoods. A key shift toward creating a child-friendly culture involves not only recognizing children's rights to safe and healthy environments for play, but accepting that we all share responsibility for creating community environments that support children's interests and well-being.

\section{Acknowledgments}

The authors would like to acknowledge the funding agencies who provided support for this study including the Canadian Institutes of Health Research, the Children's Health Foundation, the Children's Health Research Institute, Heart and Strike Foundation of Canada and the Social Sciences and Humanities Research Council of Canada. We would also like to thank the staff and students of the Thames Valley District School Board and the London District Catholic School Board for their participation. Finally, we would like to thank the staff and students of the Human Environments Analysis Laboratory at Western University, particularly Sandra Kulon and Martin Healy.

\section{Declaration of Conflicting Interests}

The author(s) declared no potential conflicts of interest with respect to the research, authorship, and/or publication of this article.

\section{Funding}

The author(s) received no financial support for the research, authorship, and/or publication of this article.

\section{Note}

1. The names of all people and locations in this study have been changed to protect the privacy of the participants.

\section{References}

Aarts, Marie-Jeanne, Sanne I. de Vries, Hans Am M van Oers, and Albertine J. Schuit. 2012. "Outdoor Play among Children in Relation to Neighborhood Characteristics: A Cross-Sectional Neighborhood Observation Study." The International Journal of Behavioral Nutrition and Physical Activity 9: Article 98. doi:10.1186/1479-5868-9-98.

Active Healthy Kids Canada. 2012. "Is Active Play Extinct? The Active Healthy Kids Canada 2012 Report Card on Physical Activity for Children and Youth." Toronto, ON: Active Healthy Kids Canada.

Aitken, Stuart C. 2001. "Global Crises of Childhood: Rights, Justice and the Unchildlike Child." Area 33 (2): 119-27. doi:10.1111/1475-4762.00015.

Bessell, Sharon, and Jan Mason. 2014. Putting the Pieces in Place: Children, Communities and Social Capital in Australia. Canberra: Australian National University.

Bishop, Kate and Linda Corkery, eds. 2017. Designing Cities with Children and Young People: Beyond Playgrounds and Skate Parks. New York: Routledge. 
Bjorklid, Pia, and Maria Nordstrom. 2007. "Environmental ChildFriendliness: Collaboration and Future Research." Children, Youth \& Environments 17 (4): 388-401.

Buliung, Ron N., Raktim Mitra, and Guy Faulkner. 2009. "Active School Transportation in the Greater Toronto Area, Canada: An Exploration of Trends in Space and Time (19862006)." Preventive Medicine 48 (6): 507-12. doi:10.1016/j. ypmed.2009.03.001.

Burdette, Hillary L., and Robert C. Whitaker. 2005a. "A National Study of Neighborhood Safety, Outdoor Play, Television Viewing, and Obesity in Preschool Children." Pediatrics 116 (3): 657-62.

Burdette, Hillary L., and Robert C. Whitaker. 2005b. "Resurrecting Free Play in Young Children Looking beyond Fitness and Fatness to Attention, Affiliation, and Affect." Archives of Pediatrics and Adolescent Medicine 159 (1): 46-50.

Carver, Alison, Anna Timperio, and David Crawford. 2008. "Perceptions of Neighborhood Safety and Physical Activity among Youth: The CLAN Study." Journal of Physical Activity and Health 5:430-44.

Chawla, Louise. 2002. "Toward Better Cities for Children and Youth." In Growing up in an Urbanising World, edited by Louise Chawla, Vol. 13, 291-42. London: Earthscan Publications.

Children's Society. 2007. "Reflections on Childhood: Friendship." London, England: Children's Society.

Christian, Hayley, Stephen J. Ball, Stephen R. Zubrick, Sally Brinkman, Gavin Turrell, Bryan Boruff, and Sarah Foster. 2017. "Relationship between the Neighbourhood Built Environment and Early Child Development." Health \& Place 48 (Suppl. C): 90-101. doi:10.1016/j.healthplace.2017.08.010.

Churchman, Arza. 2003. "Is There a Place for Children in the City?" Journal of Urban Design 8 (2): 99-111. doi:10.1080/ 13574800306482 .

City of London. 2016. "City of London-Community Profiles: Policy and Planning Support, Neighbourhood, Children and Fire Services." London, ON.

Collins, Damian C. A., and Robin A. Kearns. 2001. "Under Curfew and under Siege? Legal Geographies of Young People.” Geoforum 32 (3): 389-403. doi:10.1016/S00167185(00)00033-6.

Cosco, Nilda, and Robin Moore. 2002. "Our Neighborhood Is like That." In Growing up in an Urbanizing World, edited by Louise Chawla, 35-56. London, England: Earthscan Publications.

Derr, Victoria, Louise Chawla, and Mara Mintzer. 2018. Placemaking with Children and Youth. New York: New Village Press.

Driskell, David. 2002. Creating Better Cities with Children and Youth: A Manual for Participation. London: Earthscan.

Fyhri, Aslak, Randi Hjorthol, Roger Mackett, Trine Nordgaard Fotel, and Marketta Kyttä. 2011. "Children's Active Travel and Independent Mobility in Four Countries: Development, Social Contributing Trends and Measures." Transport Policy 18 (5): 703-10. doi:10.1016/j.tranpol.2011.01.005.

Gaster, Sanford. 1991. "Urban Children's Access to Their Neighborhood." Environment and Behavior 23 (1): 70-85. doi:10.1177/0013916591231004.

Gibson, James J. 1979. The Ecological Approach to Visual Perception. Boston: Haughton Mifflin.
Gill, Tim. 2007. No Fear: Growing up in a Risk Averse Society. London, England: Calouste Gulbenkian Foundation.

Gleeson, Brendan, and Neil Sipe, eds. 2006. Creating ChildFriendly Cities: Reinstating Kids in the City. London, England: Routledge.

Gray, Peter. 2011. "The Decline of Play and the Rise of Psychopathology in Children and Adolescents." American Journal of Play 3 (4): 443-63. doi:10.1016/B978-1-41604689-9.00030-9.

Haikkola, Lotta, Maria Giuseppina Pacilli, Liisa Horelli, and Miretta Prezza. 2007. "Interpretations of Urban ChildFriendliness: A Comparative Study of Two Neighborhoods in Helsinki and Rome." Children, Youth \& Environments 17 (4): 319-51.

Hillman, Mayer, John Adams, and John Whitelegg. 1990. One False Move. London, England: Policy Studies Institute.

Hofferth, Sandra L., and John F. Sandberg. 2001. "Changes in American Children's Time, 1981-1997." In Children at the Millenium: Where Have We Come from, Where Are We Going? edited by Sandra L. Hofferth and John F. Sandberg, Vol. 6, 193-229. Oxford, England: Elsevier Science.

Horelli, Liisa. 2007. "Constructing a Theoretical Framework for Environmental Child-Friendliness." Children, Youth \& Environments 17 (1): 267-92.

Jack, Gordon. 2008. "Place Matters: The Significance of Place Attachments for Children's Well-Being." British Journal of Social Work 40 (3): 755-71. doi:10.1093/bjsw/ben142.

Karsten, Lia. 2002. "Mapping Childhood in Amsterdam: The Spatial and Social Construction of Children's Domains in the City." Tijdschrift Voor Economische En Sociale Geografiel Journal of Economic \& Social Geography 93 (3): 231-41. doi:10.1111/1467-9663.00199.

Karsten, Lia. 2005. "It All Used to Be Better? Different Generations on Continuity and Change in Urban Children's Daily Use of Space." Children's Geographies 3 (3): 275-90. doi:10.1080/14733280500352912.

Knoll, Martin, and Jennifer Roe. 2017. "Ten Questions concerning a New Adolescent Health Urbanism." Building and Environment 126:496-506. doi:10.1016/j.buildenv.2017.10.006.

Kyttä, Marketta. 2002. “Affordances of Children's Environments in the Context of Cities, Small Towns, Suburbs and Rural Villages in Finland and Belarus." Journal of Environmental Psychology 22 (1-2): 109-23. doi:10.1006/jevp.2001.0249.

Kyttä, Marketta. 2003. Children in Outdoor Contexts: Affordances and Independent Mobility in the Assessment of Environmental Child Friendliness. Espoo, Finland: Helsinki University of Technology.

Kyttä, Marketta. 2004. “The Extent of Children's Independent Mobility and the Number of Actualized Affordances as Criteria for Child-Friendly Environments." Journal of Environmental Psychology 24 (2): 179-98. doi:10.1016/ S0272-4944(03)00073-2.

Kyttä, Marketta, Jukka Hirvonen, Julie Rudner, Iiris Pirjola, and Tiina Laatikainen. 2015. "The Last Free-Range Children? Children's Independent Mobility in Finland in the 1990s and 2010s." Journal of Transport Geography 47:1-12. doi:10.1016/j.jtrangeo.2015.07.004.

Layard, Richard, and Judy Dunn. 2009. A Good Childhood: Searching for Values in a Competitive Age. London, England: Penguin Books. 
Loebach, Janet, and Jason Gilliland. 2010. "Child-Led Tours to Uncover Children's Perceptions and Use of Neighborhood Environments" Children, Youth \& Environments 20 (1): 52-90. https://www.jstor.org/stable/10.7721/chilyoutenvi.20.1.0052.

Loebach, Janet, and Jason Gilliland. 2016a. "Free Range Kids? Using GPS-Derived Activity Spaces to Examine Children's Neighborhood Activity and Mobility." Environment and Behavior 48:421-53. doi:10.1177/0013916514543177

Loebach, Janet, and Jason Gilliland. 2016b. "Neighbourhood Play on the Endangered List: Examining Patterns in Children's Local Activity and Mobility Using GPS Monitoring and Qualitative GIS." Children's Geographies 14 (5): 573-89. doi: 10.1080/14733285.2016.1140126.

MacDougall, Colin, Wendy Schiller, and Philip Darbyshire. 2009. "What Are Our Boundaries and Where Can We Play? Perspectives from Eight to Ten Year Old Australian Metropolitan and Rural Children." Early Child Development and Care 179 (2): 189-204. doi:10.1080/ 03004430802667021.

Matthews, Hugh. 1992. Making Sense of Place: Children's Understanding of Large-Scale Environments. Hemel Hempstead, England: Harvester Wheatsheaf.

Mikkelsen, Miguel Romero, and Pia Christensen. 2009. "Is Children's Independent Mobility Really Independent? A Study of Children's Mobility Combining Ethnography and GPS/Mobile Phone Technologies." Mobilities 4 (1): 37-58. doi:10.1080/17450100802657954.

Mitchell, Christine A., Andrew F. Clark, and Jason A. Gilliland. 2016. "Built Environment Influences of Children's Physical Activity: Examining Differences by Neighbourhood Size and Sex." International Journal of Environmental Research and Public Health 13 (1): 130. doi:10.3390/ijerph13010130.

Moore, Robin. 1986. Childhood's Domain: Play and Place in Child Development. Kent, England: Croom Helm.

Osborne, Caroline, Claudia Baldwin, Dana Thomsen, and Geoffrey Woolcock. 2017. "The Unheard Voices of Youth in Urban Planning: Using Social Capital as a Theoretical Lens in Sunshine Coast, Australia." Children's Geographies 15 (3): 349-61. doi:10.1080/14733285.2016.1249822.

Play England. 2008. "Playday 2008 Opinion Poll Summary.” London, England. http://www.playday.org.uk/resources/research/2008 -research/.

Pooley, Colin G., Jean Turnbull, and Mags Adams. 2005. "The Journey to School in Britain since the 1940s: Continuity and Change." Area 37 (1): 43-53.

Rasmussen, Kim 2004. "Places for Children-Children's Places." Childhood 11 (2): 155-73.

Riggio, Eliana. 2002. "Child Friendly Cities: Good Governance in the Best Interests of the Child." Environment \& Urbanization 14 (2): 45-58.

Rissotto, Antonella, and M. Vittoria Giuliani. 2006. "Learning Neighbourhood Environments: The Loss of Experience in a Modern World." In Children and Their Environments: Learning, Using and Designing Spaces, edited by Christopher Blades and Mark Spencer, 75-90. Cambridge: Cambridge University Press.

Rissotto, Antonella, and Francesco Tonucci. 2002. "Freedom of Movement and Environmental Knowledge in Elementary School Children." Journal of Environmental Psychology 22 (1): $65-77$.
Shaw, Ben, Ben Watson, Bjorn Frauendienst, Andreas Redecker, Tim Jones, and Mayer Hillman. 2013. "Children's Independent Mobility: A Comparative Study in England and Germany (1971-2010)." London, England: Policy Studies Institute, University of Westminster.

Spencer, Christopher, and Helen Woolley. 2000. "Children and the City: A Summary of Recent Environmental Psychology Research." Child: Care, Health and Development 26 (3): 181-97. http://www.ncbi.nlm.nih.gov/pubmed/10921437.

Spilsbury, James. 2005. “We Don'T Really Get To Go Out in the Front Yard'-Children's Home Range and Neighborhood Violence." Children's Geographies 3 (1): 79-99. doi:10.1080/14733280500037281.

Talen, Emily, and Mary Coffindaffer. 1999. "The Utopianism of Children: An Empirical Study of Children's Neighborhood Design Preferences." Journal of Planning Education and Research 18 (4): 321-31. doi:10.1177/0739456x9901800404.

Tillmann, Suzanne, Andrew F. Clark, and Jason A. Gilliland. 2018. "Children and Nature: Linking Accessibility of Natural Environments and Children's Health-Related Quality of Life." International Journal of Environmental Research and Public Health 15 (6): 1072. doi:10.3390/ijerph15061072

Valentine, Gill. 1996. "Angels and Devils: Moral Landscapes of Childhood." Environment and Planning D 14:581-600.

Valentine, Gill. 1997. “'Oh Yes I Can' 'Oh No You Can't': Children and Parents' Understandings of Kids' Competence to Negotiate Public Space Safely." Antipode 29 (1): 65-89. doi:10.1111/1467-8330.00035.

Valentine, Gill, and John McKendrick. 1997. "Children's Outdoor Play: Exploring Parental Concerns about Children's Safety and the Changing Nature of Childhood." Geoforum 28 (2): 219-35.

Villanueva, Karen, Hannah Badland, Amanda Kvalsvig, Meredith O'Connor, Hayley Christian, Geoffrey Woolcock, Billie GilesCorti, and Sharon Goldfeld. 2016. "Can the Neighborhood Built Environment Make a Difference in Children's Development? Building the Research Agenda to Create Evidence for PlaceBased Children's Policy." Academic Pediatrics 16 (1): 10-19. doi:10.1016/j.acap.2015.09.006.

Villanueva, Karen, Billie Giles-Corti, Max Bulsara, Gavin R. McCormack, Anna Timperio, Nick Middleton, Bridget Beesley, and Georgina Trapp. 2012. "How Far Do Children Travel from Their Homes? Exploring Children's Activity Spaces in Their Neighborhood." Health \& Place 18 (2): 263-73. http://www. sciencedirect.com/science/article/pii/S1353829211001936.

Villanueva, Karen, Billie Giles-Corti, Max Bulsara, Anna Timperio, Gavin R. McCormack, Bridget Beesley, Georgina Trapp, and Nicholas Middleton. 2012. "Where Do Children Travel to and What Local Opportunities Are Available? The Relationship between Neighborhood Destinations and Children's Independent Mobility." Environment and Behavior 45 (6): 679-705.

Wilson, Katherine, Andrew F. Clark, and Jason A. Gilliland. 2018. "Understanding Child and Parent Perceptions of Barriers Influencing Children's Active School Travel." BMC Public Health 18: Article 1053. doi:10.1186/s12889-018-5874-y.

Woolley, Helen. 2006. "Freedom of the City: Contemporary Issues and Policy Influences on Children and Young People's Use of Public Open Space in England." Children's Geographies 4 (1): 45-59. doi:10.1080/14733280600577368. 
Woolley, Helen, Jessica Dunn, Christopher Spencer, Tania Short, and Gwyn Rowley. 1999. "Children Describe Their Experiences of the City Centre: A Qualitative Study of the Fears and Concerns Which May Limit Their Full Participation." Landscape Research 24 (3): 287-301. doi:10.1080/01426399908706564.

\section{Author Biographies}

Janet Loebach (BEngSoc, MEnvDesign, PhD, PEng) is an environmental design researcher and consultant based in Ontario, Canada. Her research and practice focuses on children's perception and use of their everyday environments, and the socioenvironmental factors that influence or support children's behavior and wellbeing. She also has extensive experience with participatory, childled, and community-based planning processes.

Jason Gilliland (BAHon, MA, MArch, $\mathrm{PhD}$ ) is a director of the Urban Development Program and full professor in the Department of Geography, Department of Pediatrics, School of Health Studies, and Department of Epidemiology and Biostatistics at Western University. His research is primarily focused on exploring social and environmental determinants and developing interventions to ameliorate children's health issues, such as poor nutrition, physical inactivity, and obesity. 